\title{
INVESTIGATIONS ON ZnSe/GaAs INTERFACE BY TREATMENT OF GaAs $(2 \times 4)$ SURFACE WITH Te AND Mg
}

\author{
R. Ebel, W. Spain, H.R. Ress, D. Albert, H. Schäfer, M. Ehinger, \\ W. Faschinger and G. Landwehr \\ Physikalisches Institut der Universität Würzburg \\ Am Hubland, 97074 Würzburg, Germany
}

\begin{abstract}
The heterovalent interface $\mathrm{ZnSe} / \mathrm{GaAs}$, despite the small lattice misfit, still poses certain problems. The condition of the substrate surface prior to growth start determines the initial growth conditions, which on the other hand are assumed to be responsible for defect densities. Since $\mathrm{Zn}$, in contrast to Se, hardly binds to GaAs the initial surface during growth start is essentially Se terminated. Therefore the binding of $\mathrm{Mg}$ to Se terminated GaAs was investigated. The structural quality of $140 \mathrm{~nm}$ thick $\mathrm{ZnSe}$ layers on different $\mathrm{MgSe}$ coverages were compared to conventionally grown and $\mathrm{Te}$ initiated $\mathrm{ZnSe}$ epilayers of the same thickness.
\end{abstract}

PACS numbers: $68.55 . \mathrm{Bd}, 68.65 .+\mathrm{g}$

\section{Introduction}

Due to the small lattice mismatch between GaAs and $\mathrm{ZnSe}$, GaAs is the dominant substrate material for MBE growth of blue-green optoelectronic II-VI devices. Nevertheless the heterovalent interface still remains a major problem.

The initial growth conditions determine the defect densities [1], which on the other hand are known to be responsible for the degradation of optoelectronic devices [2]. Therefore a precise control of the initial surface is of great importance. Since the sticking coefficient of $\mathrm{Zn}$ on GaAs is much smaller than that of Se, $\mathrm{ZnSe}$ growth starts on a Se terminated GaAs surface (Se/GaAs) if $\mathrm{Zn}$ and Se are offered simultaneously. This surface has been investigated [1] and is known to be almost inert [3]. Therefore ZnSe growth proceeds predominantly at defects, which results in an unfavorable island growth mode and high defect densities [4].

One obvious way to avoid the Se termination of GaAs is the exposure of the $(2 \times 4) \mathrm{GaAs}$ surface to $\mathrm{Zn}$ prior to $\mathrm{ZnSe}$ growth. Compared to conventionally grown $\mathrm{ZnSe}$ the defect density is significantly reduced [5]. However, since $\mathrm{Zn}$ does not stick on $(2 \times 4)$ GaAs [6], this approach has the disadvantage to rely on the reaction dynamics. Recently we have demonstrated that similar improvement can 
be obtained by a static approach, when $\mathrm{ZnSe}$ growth is started on a GaAs surface terminated with $\mathrm{Te}$ [6]. This procedure leads to a two-dimensional growth mode already during the first few monolayers, resulting in a generally improved layer quality and a significantly higher critical layer thickness [6].

In this paper we investigate an alternative static growth start, i.e. the nucleation of $\mathrm{Mg}$ on $\mathrm{Se} / \mathrm{GaAs}$. Since the bonding strength of $\mathrm{Mg}$ to Se is higher than $\mathrm{Zn}$ to Se, $\mathrm{Mg}$ should be able to bond to Se terminated GaAs. This surface as well as the $\mathrm{ZnSe}$ growth start on such $\mathrm{Mg}$ treated surfaces was studied by X-ray photoelectron spectroscopy (XPS) and reflection high energy electron diffraction (RHEED). The structural quality of $\mathrm{ZnSe}$ layers on $\mathrm{Mg}$ terminated Se/GaAs was controlled by high resolution X-ray diffraction (HRXRD).

\section{Experimental set up}

All samples were prepared on GaAs buffer layers which were grown in a III/V-growth chamber with a background pressure less than $2 \times 10^{-10}$ mbar. The substrate temperature was controlled by a thermocouple. Standard effusion cells were used to evaporate $\mathrm{Ga}, \mathrm{As}_{4}$ and $\mathrm{Si}$. The flux was determined by an ion gauge, measuring the beam equivalent pressure (BEP). The surface symmetry was observed by RHEED (12 kV acceleration voltage).

The samples were subsequently moved to the II/VI-growth chamber under UHV conditions (pressure smaller than $5 \times 10^{-10} \mathrm{mbar}$ ). Standard effusion cells ( $\mathrm{Zn}, \mathrm{Se}, \mathrm{ZnCl}, \mathrm{Mg}$ and $\mathrm{Te}$ ) were employed and the flux measurement was similar to that in the III/V-growth chamber. The surface reconstruction and topology was observed by RHEED (30 kV).

XPS measurements were performed in a separate surface analysis chamber which is also connected to the growth chambers via an UHV transfer module. $\mathrm{X}$-rays were provided by an $\mathrm{Al} K_{\alpha}(1486.6 \mathrm{eV})$ source. The resolution of the energy analyzer was $0.3 \mathrm{eV}$.

A high-resolution six crystal-diffractometer equipped with a laboratory X-ray source $\left(\mathrm{Cu} K_{\alpha}\right)$ provided the information about the structural quality of the grown ZnSe layers. The photoluminescence measurements were performed in a helium bath cryostat at $2 \mathrm{~K}$ with a HeCd laser at a wavelength of $325 \mathrm{~nm}$ with an optical power of $3 \mathrm{~mW}$.

\section{Substrate and surface preparation}

The substrates were first cleaned under nitrogen flow, then etched in a $\mathrm{NH}_{4} / \mathrm{H}_{2} \mathrm{O}_{2}$ solution, rinsed with water and finally dried under nitrogen flow. They were glued to the molybdenum holders with indium.

The oxide layer of the GaAs substrate was desorbed under As-flux at $580^{\circ} \mathrm{C}$. The $250 \mathrm{~nm}$ thick silicon doped GaAs buffer layers were grown at $600^{\circ} \mathrm{C}$ with a growth rate of $500 \mathrm{~nm} / \mathrm{h}$ under As-rich conditions (As BEP $\approx 2.5 \times 10^{-5} \mathrm{mbar}$, Ga BEP $\approx 5.0 \times 10^{-7} \mathrm{mbar}$ ). The flux of the silicon oven at $1100^{\circ} \mathrm{C}$ provided a doping concentration of $5 \times 10^{18} \mathrm{~cm}^{-3}$. The growth was stopped by leaving the samples under As flux for two minutes, subsequently closing the shutter and simultaneously turning off the substrate heater. This leads to a $(2 \times 4)$ symmetry as observed by RHEED. 
This pattern was still observable after transfer at room temperature to the II/VI-growth chamber. The samples were again brought to a substrate temperature of $300^{\circ} \mathrm{C}$. To obtain the initial interfaces, we exposed the specimens to Se (BEP $\left.2.5 \times 10^{-6} \mathrm{mbar}\right)$ for two minutes. The appearing $(2 \times 1)$ reconstruction of Se terminated $\mathrm{GaAs}$ (Se/GaAs) is a relatively well-known surface [1]. It is generally assumed that half of the Se absorbed remains at the surface, whereas the other half is incorporated into underlying layers [3]. The Se/GaAs surface was then exposed to a $\mathrm{Mg}$ flux (BEP $1.0 \times 10^{-8} \mathrm{mbar}$ ), which led to a $(1 \times 1)$ reconstruction pattern.

We investigated the nucleation of $\mathrm{Mg}$ on $\mathrm{Se} / \mathrm{GaAs}$ as a function of the time of exposure. For this purpose the sample was exposed to a constant Mg flux (BEP $1.0 \times 10^{-8} \mathrm{mbar}$ ). The various coverages were examined by XPS. Therefore, the samples had to be moved to the surface analysis chamber (background pressure smaller than $3 \times 10^{-11} \mathrm{mbar}$ ) at room temperature for the measurement and afterwards back to the growth chamber, where they had to be brought to $300^{\circ} \mathrm{C}$ again. A special care was taken to avoid contamination between successive exposures. This included the observation of carbon and oxygen peaks at every measurement. None of the two was observed in a crucial amount.

We examined the low binding energy range of the concerned elements by XPS (Ga 3d, Se 3d, As 3d and Mg 2s), all peaks lying between 0 and $100 \mathrm{eV}$. Therefore the photoelectrons all have about the same attenuation length which is necessary to be comparable. Because of the diminishing intensity of the Mg $2 s$ peak, we also investigated the $\mathrm{Mg} 1 s$ peak at $1305 \mathrm{eV}$. The peak intensities were obtained by subtracting the linear background, integrating over the peak area and multiplying each peak by its cross-section. Each intensity was normalized to the total sum of all peaks, the latter serving as a measure of the X-ray photon flux. A scale factor of the $\mathrm{Mg} 1 s$ peak was obtained by multiplying the ratio of the counting rates of $\mathrm{Mg} 2 s$ to $\mathrm{Mg} 1 s$ by the cross-section of $\mathrm{Mg} 2 s$ for a reasonably high surface coverage. Subsequently the growth start of $\mathrm{ZnSe}$ on the $\mathrm{Mg} / \mathrm{Se} / \mathrm{GaAs}$ surface was observed by RIIEED. We studied the growth on three different $\mathrm{Mg}$ coverages, namely $0.5,1$ and 2 minutes. As deduced from a $\mathrm{MgSe}$ growth rate under Se rich conditions this corresponds to coverages of approximately $0.75,1.5$ and 3 monolayers (ML).

For each $\mathrm{Mg}$ coverage a doped and an undoped $\mathrm{ZnSe}$ layer of $140 \mathrm{~nm}$ was grown (Zn BEP $5 \times 10^{-7} \mathrm{mbar}$ ). For low $\mathrm{Mg}$ coverage the RHEED pattern exhibited a two-dimensional growth after 30 seconds, whereas for two monolayers an island growth mode persists for almost a period of two minutes.

\section{Results and discussion}

XPS measurements reveal a linearly increasing surface concentration of $\mathrm{Mg}$ for the first minute (Fig. 1). The coverage can reach even more than one monolayer. Therefore $\mathrm{Mg}$ binds to Se/GaAs but does not reach saturation. For exposure times longer than 120 seconds a second $\mathrm{Mg} 2 s$ peak appears which is shifted by $0.9 \mathrm{eV}$. This chemical shift can be interpreted as the formation of metallic $\mathrm{Mg}$ in addition to the $\mathrm{Mg}$ bond to $\mathrm{Se} / \mathrm{GaAs}$. The component of the $\mathrm{Mg}-\mathrm{Se} / \mathrm{GaAs}$ binding remains approximately constant, whereas the further increase in the total $\mathrm{Mg}$ signal is completely due to the metallic component. Therefore, in contrast to Te which 


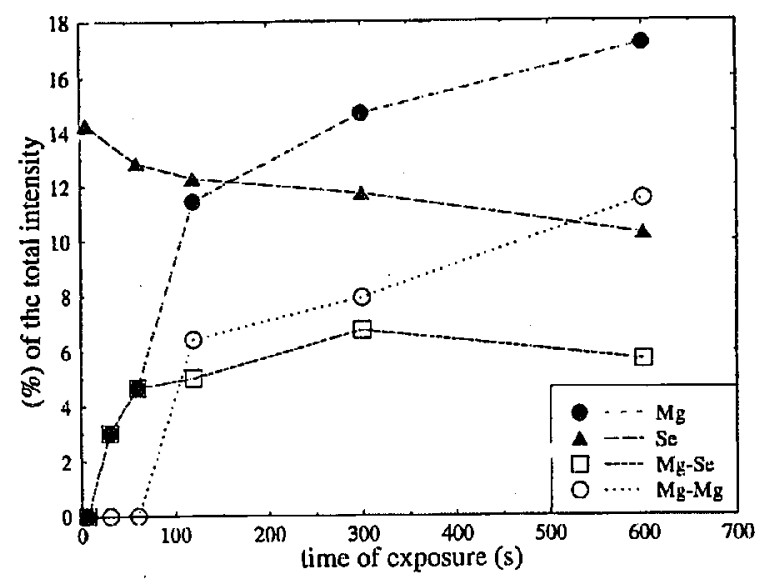

Fig. 1. Surface concentration of magnesium and selenium as a function of time of exposure. The unfilled markers show the contribution of the $\mathrm{Mg}-\mathrm{Se}$ and $\mathrm{Mg}-\mathrm{Mg}$ components.

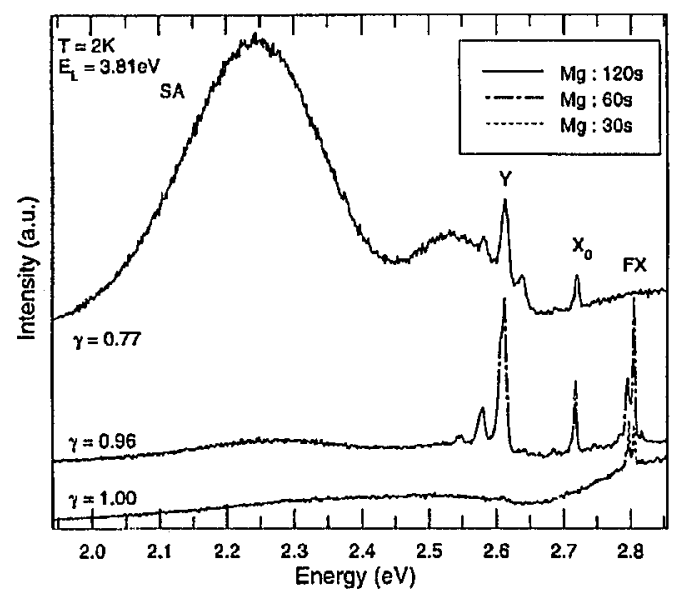

Fig. 2. Photoluminescence spectrum of $140 \mathrm{~nm}$ undoped $\mathrm{ZnSe}$ on different $\mathrm{Mg}$ coverages. $\gamma$ is the strain parameter depicting the degree of relaxation.

saturates after 0.5 monolayers, the amount of $\mathrm{Mg}$ deposited may influence the growth start.

This is confirmed by photoluminescence measurements on undoped $\mathrm{ZnSe}$ layers grown on different $\mathrm{Mg}$ coverages (Fig. 2). For higher $\mathrm{Mg}$ coverages the defect emission increases drastically, whereas for the low $\mathrm{Mg}$ coverage almost no photoluminescence is observed. A good optical quality is only obtained for a $\mathrm{Mg}$ coverage around one monolayer.

Figure 3 compares a PL spectrum of a doped sample grown directly on $\mathrm{Se} / \mathrm{GaAs}$ with the one of a sample grown on $\mathrm{Mg} / \mathrm{Se} / \mathrm{GaAs}$ with optimized $\mathrm{Mg}$ 


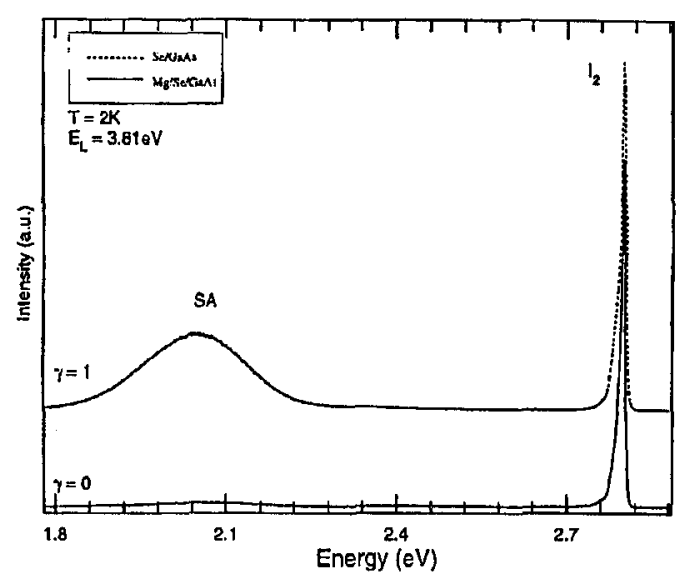

Fig. 3. Comparison of $180 \mathrm{~nm}$ doped $\mathrm{Se} / \mathrm{GaAs}$ and $240 \mathrm{~nm}$ doped $\mathrm{Mg} / \mathrm{Se} / \mathrm{GaAs}$ with optimised $\mathrm{Mg}$ coverage.

coverage. The spectrum of the layer grown on $\mathrm{Mg} / \mathrm{Se} / \mathrm{GaAs}$ shows a significantly lower ratio of the excitonic to defect emission compared to $\mathrm{ZnSe} / \mathrm{Se} / \mathrm{GaAs}$ layers. As we have shown previously [4] this indicates an improved interface quality.

By using IIRXRD the strain parameter and the structural quality of the samples was determined. If the epilayer is pseudomorphic the 004-rocking curves show oscillations from which the layer thickness can be determined. The position of the epilayer peak relative to the substrate peak depends on the lattice constant and thus is a measure of the relaxation. The relaxation of the $\mathrm{Mg}$ initiated $\mathrm{ZnSe}$ layers was compared to conventionally grown and Te initiated ZnSe layers of the same thicknesses.

For low $\mathrm{Mg}$ coverage $(0.75 \mathrm{ML})$ the $140 \mathrm{~nm} \mathrm{ZnSe}$ layers are fully strained, whereas for higher coverages the layers of the same thickness are partially relaxed. The $\mathrm{Mg}$ seems to be incorporated into the compound as a $\mathrm{Mg}(\mathrm{Zn})$ Se layer and thus introduces additional strain. MgSe has a larger lattice constant than $\mathrm{ZnSe}$ and GaAs and thus increases the compressive strain.

One measure for the structural quality of epilayers is the critical thickness of pseudomorphic grown layers which is determined by the defect density and the built-in strain. Since the additional strain introduced by the MgSe layer leads to an earlier relaxation we calculated the stored strain. According to the Hooke law the strain energy stored in a fully or partially coherent epitaxial layer is proportional to the layer thickness and the square of the lattice displacement parallel to the interface. For multilayers, the total energy is simply the sum of the strain energy for each layer [7].

We therefore calculated the energy stored in $140 \mathrm{~nm} \mathrm{ZnSe}$ grown on the above described MgSe interfaces. The Mg initiated epitaxial layers can sustain about the same amount of strain energy as normally grown layers, but significantly less than those initiated with Te coverage. 


\section{Conclusions}

The growth of ZnSe starts, due to the small sticking coefficient of $\mathrm{Zn}$, on a Se terminated GaAs surface. Since Zn can only bind to defects, an island growth mode results, leading to a high stacking fault density.

We investigated the nucleation of $\mathrm{Mg}$ on Se terminated GaAs to improve the initial growth mode. In contrast to $\mathrm{Zn}, \mathrm{Mg}$ sticks to Se/GaAs, according to XPS measurements. We were able to show that the structural quality of epitaxial $\mathrm{ZnSe}$ layers on different $\mathrm{MgSe}$ coverages is as good as conventionally grown layers, whereas the optical quality increases. Nevertheless the Te initiated layers possess a significantly higher structural quality.

\section{References}

[1] M.D. Pashley, D. Li, J. Vac. Sci. Technol. A 12, 1884 (1994).

[2] A. Ishibashi, IEEE J. Sel. Topics Quantum Electron. 1, 741 (1995).

[3] M.D. Pashley, D. Li, Proc. SPIE 2346, 142 (1994).

[4] S. Guha, H. Munekata, L.L. Chang, J. Appl. Phys. 73, 2294 (1993).

[5] S. Saito, P.J. Parkbrook, S. Nakamura, Y. Nishikawa, G. Hatakoshi, in: Proc. Intern. Symposium on Blue Laser and Light Emitting Diodes, Chiba 1996, Eds. A. Yoshikawa, K. Kishino, M. Kobayashi, T. Zasuda, Ohmsha Ltd, Tokyo 1996, p. 66 .

[6] W. Spahn, H.R. Reß, K. Schüll, M. Ehinger, D. Hommel, G. Landwehr, J. Cryst. Growth 159, 761 (1996).

[7] J.Y. Tsao, in: Materials Fundamentals of Molecular Beam Epitaxy, Ed. H.B. Jovanovich, Academic Press, Boston 1993. 